\title{
Charge-transfer plasmons with narrow conductive molecular bridges: A quantum-classical theory
}

\author{
A.S. Fedorov, 1, 2, 3, a) P.O. Krasnov, ${ }^{2,3,4}$ M.A. Visotin, ${ }^{1,3}$ F.N. Tomilin, ${ }^{1,3}$ S.P. Polyutov, ${ }^{1,3, \text { b) }}$ and H. Agren ${ }^{2,5}$ \\ 1) Kirensky Institute of Physics, Federal Research Center KSC SB RAS, 660036 Krasnoyarsk, \\ Russia \\ 2) Federal Siberian Research Clinical Center under FMBA of Russia, 660037 Krasnoyarsk, \\ Russia \\ ${ }^{3)}$ Siberian Federal University, 660041 Krasnoyarsk, Russia \\ 4) Reshetnev Siberian State University of Science and Technology, 660037 Krasnoyarsk, \\ Russia \\ ${ }^{5)}$ Division of Theoretical Chemistry and Biology, Royal Institute of Technology, SE-100 44 Stockholm, \\ Sweden
}

We analyze a new type of plasmon systems arising in small metal nanoparticles linked by narrow conductive molecular bridges. In contrast to the well-known charge-transfer plasmons, the bridge in these systems consists only of a narrow conductive molecule or polymer in which the electrons move in a ballistic mode, showing quantum effects. The plasmonic system is studied by an original hybrid quantum-classical model accounting for the quantum effects, with the main parameters obtained from first-principle DFT simulations. We have derived a general analytical expression for the modified frequency of the plasmons and have shown that its frequency lies in the near-infrared region and strongly depends on the conductivity of the molecule, on the nanoparticle - molecule interface and on the size of the system. As illustration, we explored the plasmons in a system consisting of two small gold nanoparticles linked by a conjugated polyacetylene molecule terminated by sulfur atoms. It is argued that applications of this novel type of plasmons may have wide ramifications in the areas of chemical sensing and IR deep tissue imaging.

Keywords: Charge-transfer plasmons, Density Functional Theory, Nanoparticles

\section{INTRODUCTION}

Surface plasmons (SPs) are delocalized collective oscillations of free electrons relative to the positive ions at the interface between negative and positive permittivity materials, like metal-dielectric interfaces. SPs are associated with oscillations of charge density coupled with electromagnetic fields created by coherent motion of the free charges. These oscillations are called surface plasmon polaritons in the case of planar interfaces or localized surface plasmons (LSPs) in the case of metal nanoparticles with a closed surface ${ }^{1,2}$. The plasmon polaritons are characterized by a specific frequency (surface resonant frequency, SRF), when the incident electromagnetic radiation is resonant with the surface plasmon resonance (SPR) frequency. The SRF is highly sensitive to the permittivity of a surrounding chemical environment, the change of which due to chemical composition and morphology often leads to substantial shifts of the $\mathrm{SRF}^{3-6}$.

Since the SRF can be readily measured by different optical techniques ${ }^{3}$, LSPs are nowadays intensively used for the creation of nanoscale sensors for chemical and biological substances ${ }^{4-7}$. Moreover, the strong local electromagnetic field enhancements offered by plasmon excitation has rendered a wide use of plasmonic materials in different fields, such as high resolution imaging ${ }^{8}$, plasmon lasers ${ }^{9}$, chemical synthesis ${ }^{10}$, water splitting ${ }^{11}$, optical waveguiding ${ }^{12-15}$, biomedical and telecom applications ${ }^{16,17}$ and photovoltaic cells $^{1,18-21}$, just to name a few.

\footnotetext{
a)Electronic mail: alex99@iph.krasn.ru

b)Electronic mail: spolyutov@sfu-kras.ru
}

Gold is the most known substance for plasmonic applications due to its light absorption in the visible region. It is often used in the form of separate nanoparticles (NPs), nanorods or 3-D materials built from NPs ${ }^{22,23}$. Besides of Au NPs, silver and copper nanoparticles are also employed, albeit to a lesser extent due to their instability $1,4,7,8,10,19,21$. A variety of different forms of plasmonic materials is conditioned by the fact that the LSP is concentrated at the edges of the nanoparticles, that the strength of the electromagnetic field strongly depends on the interaction and the interparticle distance, and that small changes are very influential on the measured signal. From this point of view, the SRF also depends on the size and shape of the nanoparticles ${ }^{1-6}$ as well as type of material that links them ${ }^{5}$. In the case of conductive materials between two nanoparticles a new mode, called charge transfer plasmon (CTP), emerges. Such plasmons, observed in the case of conductively coupled metallic nanoparticles, are expected to expand their applications from molecular sensing to nanoscale wave-guiding.

An example of charge transfer plasmons was experimentally observed in Ref. ${ }^{5}$ in a system consisting of two gold NPs with diameter $D \geq 40 \mathrm{~nm}$ joined by a cylindrical gold bridge with radius from 10 to $20 \mathrm{~nm}$. The CTP was described by classical Maxwell's electrodynamics as quantum effects were not expected to be significant due to the large system size. Quantum effects of CTPs have subsequently been investigated in systems consisting of two NPs separated by sub-nanometer inter-particle gaps ${ }^{5,25-27}$, where the coupling of the nanoparticles is conditioned by tunneling between them and by screening effects. However, CTPs have not been investigated for systems consisting of NPs connected by narrow conductive bridges (conducting organic molecules, COMs) where the im- 
portance of quantum effects is expected - this is precisely the topic of the present study. We thus consider here a hybrid model which exhibits CTPs consisting of two metallic NPs connected by a thin COM and analyze the implications of such systems, which thus are novel for plasmonic generation.

\section{APPLIED APPROACHES FOR CALCULATING OF ELECTRON TRANSPORT AND PLASMON OSCILLATIONS}

Generally, theoretical descriptions of plasmon resonances in bulk materials can be based on Maxwell's equations. At least, measured plasmon peaks positions and spectral areas in bulk metals are found to be in good agreement with classical theory predictions. However, surface plasmon resonance widths in conductive nanostructures are significantly influenced by quantum confinement effects. Thus, in the case of small nano-objects, they cannot be reproduced using the dielectric functions of bulk materials ${ }^{24}$.

The simplest bulk plasmon description can be obtained in terms of simple electrostatics and assuming that the electron is a classical particle having mass $m$ and coordinate $x$ as a function of time $\vec{x}(t)$

$$
m \ddot{\vec{x}}(t)=e \vec{E}=-4 \pi n e^{2} \vec{x}(t)
$$

Such an approach can, however, not be used for the here suggested NPs-COM systems, because the nature of the restoring force acting on the electron will be different and because a COM has a few conducting channels where electrons in the vicinity of the Fermi level $E_{F e r m i}$ will conduct current. Thus quantum effects of free carrier motion are vital in COMs which calls for a strict treatment by wave function time evolution $\psi(r, t)^{38}$ in the frame of the real-time propagation approaches, like real-time TDDFT ${ }^{30}$. Unfortunately, to avoid the errors accumulating in this technique the time step needs to be chosen to be very small ( $\sim 0.001 \mathrm{fs})$, which currently restricts the system size to $\sim 250$ noble ( $\mathrm{Au}, \mathrm{Ag}$ ) atoms while, as it was mentioned above, an observation of SPRs is possible only in the case of nanoparticles consisting of hundreds of atoms. Therefore it is vital to develop a methodology to calculate the plasmon frequencies in NPs-COM systems where the NP size is larger than a nanometer, while at the same time the electron current through the COM narrow bridge should be treated by a quantum-mechanical approach. The methodology should make it possible to calculate the longitudinal plasmonic frequencies in dependence of the nanoparticle diameter and also the conductive properties of the organic molecule connecting the NPs. As a first step in this methodology it is necessary to study the electronic conductivity in the systems. The Landauer method is usually used for this purpose ${ }^{41}$, according to which the effective current $I$ at zero temperature between two electrodes possessing $\mu_{1}$ and $\mu_{2}$ electrochemical potentials, respectively, can be estimated as a difference of currents from one electrode to the other one: $I=I^{+}-I^{-}$, where

$$
\begin{aligned}
I^{+} & =\frac{e}{L} \sum_{k, n} \frac{\partial E(k)}{\hbar \partial k} f\left(E, \mu_{1}\right), \\
I^{-} & =\frac{e}{L} \sum_{k, n} \frac{\partial E(k)}{\hbar \partial k} f\left(E, \mu_{2}\right) .
\end{aligned}
$$

Herein $f\left(E, \mu_{1}\right)=1 /\left(\exp \left[\left(E-\mu_{1}\right) /\left(k_{b} T\right)\right]+1\right)$ is the Fermi function of one electrode with potential $\mu_{1} ; n$ and $k$ are the indices of each transverse mode and wave number of electron state $\{n, k\}$ moving along the narrow bridge simultaneously.

Usually in the range $E(k) \in\left[\mu_{1} \ldots \mu_{2}\right]$ a fixed number $M(E)$ of the channel conducting electrons throughout the bridge is constant $M(E)=\sum_{n} f\left(E, \varepsilon_{n}\right)$. So, applying (1) and replacing the summation over $k$ by integration over $E$ the total current $I$ can be written as:

$$
\begin{gathered}
I=I^{+}-I^{-}=\frac{2 e^{2}}{h} M \frac{\left(\mu_{1}-\mu_{2}\right)}{e} \Rightarrow \\
\Rightarrow G=\frac{2 e^{2}}{h} M, 58
\end{gathered}
$$

where $G$ is the system conductivity, and $M=M\left(\mu_{1}\right)-$ $M\left(\mu_{2}\right)$. If we take into account that an electron has some reflection rate during its motion from one electrode to the other at low bias, eq.(4) would be transformed into the well-known Landauer ${ }^{42}$ formula $G=\left(2 e^{2}\right) / h * T M$, where $T=T(E)$ is the transmission coefficient at any energy $E \in\left[\mu_{1} \ldots \mu_{2}\right]$. If the bias $\left[\mu_{1} \ldots \mu_{2}\right]$ is sufficiently large so that $T(E)$ and $M(E)$ can be varied within the range, this formula can be substituted by an integral ${ }^{43}$

$$
\frac{e}{h} \int_{-\infty}^{+\infty} T(E)\left[f\left(E, \mu_{1}\right)-f\left(E, \mu_{2}\right)\right] d E
$$

which transforms at temperature $T=0$ into

$$
\frac{e}{h} \int_{\mu_{2}}^{\mu_{1}} T(E) d E
$$

Unfortunately, the Landauer approach in the original form is not profitable for a description of the proposed plasmon model because this approach does not account for the influence of the electromagnetic field (EMF) on the electron acceleration during the motion inside a narrow conductor. It proclaims the change of total electron energy instead, or, in other words, the change of electrochemical potential in the electrode. However a change of electronic state in the COM region due to the influence of an EMF is essential for the plasmon vibration description. Another reason why the Landauer approach is not profitable for our model is the assumed constant bias, while in the case of plasmon oscillations the bias and current are varied according to the harmonic law. In that context we note that, recently, some progress has been accomplished 
for AC calculations inside narrow channels ${ }^{48-50}$. For example, biased molecular junctions subjected to external timedependent electromagnetic fields have been addressed by solutions of coupled Green's functions of the quantum system and Maxwell's equations for electric and magnetic fields ${ }^{50}$. Unfortunately, this method is too complicated to use for real systems, due to the nature of the self-consistent differential equations for Green functions for electron state calculations. Also solutions of the simultaneous Maxwell's equations obtained by sampling in time and space regions using the finitedifference-time-domain (FDTD) approach is also expensive. Due to these reasons this method has been used for model systems only, where the conductive molecule is represented by a bridge in two-level systems ${ }^{50}$.

In another example ${ }^{48}$ the equation of motion for density operator $\hat{\rho}(t)$, projected onto the subspace of molecular degrees of freedom, was formulated in the weak molecule-leads coupling limit, where this junction has a time-periodic modulation of the chemical potential of the leads. For periodic timedependent terms the charge transport kinetics was mapped onto a static problem of a molecule interacting with multiple leads. The time-averaged current reproduces the results of scattering approaches to transport through conductance channels with time-dependent perturbations, as derived using the Floquet theory ${ }^{51}$. Unfortunately, this method is also too complicated to be used for real systems and is restricted to model systems only.

Time-dependent density functional theory (TDDFT) quantum method has been successfully used for description of quantum effects related to plasmons ${ }^{28-30}$. It has been shown, for example, that $\mathrm{Ag}-\mathrm{Au}$ nanoparticles are very sensitive to the chemical configuration, and in some cases the position of the atomic species outweigh the effect of changing composition ${ }^{31}$. Density Functional based Tight Binding (DFTB) methods have been used, for example, for study of optical properties and electronic structure of Ag nanorods and nanorod dimers ${ }^{54}$ and for description of influence of quantum tunneling on the efficiency of excitation energy transfer in plasmonic Ag NPs chain waveguides ${ }^{55}$. TDDFT and DFTB, being quantum methods, are, however, very time-consuming methods and the possibility of their practical use strongly depends on the size of the NPs, i.e. number of atoms and electrons. Therefore the applications are limited to the consideration of small particles, consisting, in most cases, of maximum a few hundreds atoms ${ }^{31,34,35}$.

\section{MODELING OF CHARGE-TRANSFER IN A PAIR OF METAL NANOPARTICLES BOUND BY A CONDUCTING MOLECULE}

To construct the model we have considered a system consisting of two gold nanoparticles of 147 atoms connected by a poly acetylene molecule $\mathrm{C}_{8} \mathrm{H}_{8}$, having conjugated chemical bonds, where the junctions with the NPs are implemented by the sulfur atoms to ensure the electric conductivity of the full NP-COM-NP system. Nanoparticles of such size exhibit metallic properties due to thermal excitation, see the solid electronic density of states (DOS) near $E_{\text {Fermi }}$ in Fig.2, where the gaussian broadening $0.03 \mathrm{eV}$ was used, which is comparable with the thermal broadening at temperature $300 \mathrm{~K}$. The NP metallic properties are essential for our model, where carriers move between the two nanoparticles. Herein it must be noted that the use of sulfur atoms is well-known practice for organic linker molecules to be attached to gold nanoparticles.

It is assumed that the conductive bridge is actually a onedimensional conductor in which electrons or holes move in a ballistic mode, i.e. the mean free path of the carriers exceeds the length of the bridge.

The key point of our model is the consideration of electron dynamics, which is described in the language of the wave function. Under the applied electrostatic field the conduction electrons would accelerate, which leads to change in their quasi-momentum $\vec{k}$ and the band energy $\mathrm{E}(\vec{k})$. Changes of these quantities can easily be calculated from knowledge of only the band structure and effective electron mass $m^{*}$ near $E_{\text {Fermi }}$. On the basis of so-called effective mass theorem the equation $\hbar \dot{\vec{k}}=\dot{\vec{p}}=e \vec{E}$ is proved ${ }^{36}$ that is the basic when considering the dynamics of an electron and establishing the exact relationship between quantum and classical quantities - a quasi-momentum, momentum and external force. Therefore, the method that uses the exact relationship between quantum and classical quantities is significantly easier compared to known methods based on non-equilibrium quantum Green functions ${ }^{50}$ or quantum real-time propagation of wave functions in the frame of time-dependent density functional theory (real-time TDDFT) approaches ${ }^{28-30}$. These methods can only be used for sufficiently small systems or model systems. An important assumption of the proposed model is also that the conductivity of non-periodical NP-COM-NP systems is similar to that of the corresponding periodic -(NP-COM)- systems.

This assumption can be justified by that the local dynamics of an electron inside the COM between two NPs is determined by the local electric field and that not depends strongly on the contacts of these NPs with other COMs in periodic systems. This allows us to simplify the calculations of the electron dynamics in NP-COM-NP systems and calculate the effective electron mass near $E_{F e r m i}$ only in the corresponding periodical system.

In this work calculations of the system geometry, electronic properties and band structure were carried out within density functional theory with gradient decomposition (DFT-GGA), using the VASP 5.4 software $^{39,40}$. The results of these calculations indicate that the system has metallic properties and can conduct DC or AC current, see Fig. 1, and Fig. 2.

To confirm the NP-COM-NP system static conductivity, the transmission coefficient $T(E)$, i.e the probability that an electron with energy $E$ injected at one electrode will be transmitted to the other one, was calculated in the frame of Landauer methodology using the method of Non-Equilibrium Green Functions (NEGF), which was firstly proposed in the sixties by Keldysh and others ${ }^{44},{ }^{45}$ and widely used now ${ }^{46}$. The $T(E)$ function of the polyacetylene chain $\mathrm{C}_{8} \mathrm{H}_{8} \mathrm{~S}_{2}$ connected to two gold electrodes via the sulfur atoms was calculated, see Fig.3, using the OpenMX package ${ }^{47}$. The length of the $\mathrm{C}_{8} \mathrm{H}_{8} \mathrm{~S}_{2}$ chain was equal to $11.6 \AA$. Semi-infinite one-dimensional gold 


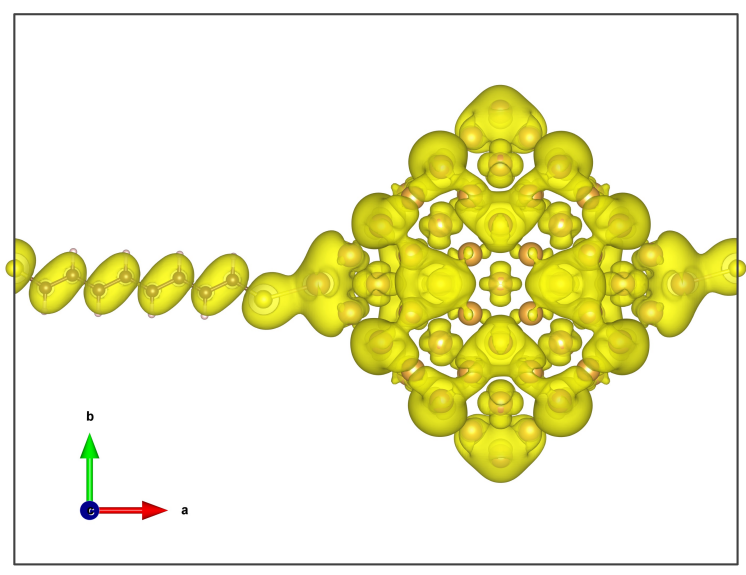

FIG. 1. Geometry and electron density of the conductive band for $k \cong k_{f}$ of periodic $-\left[\mathrm{Au}_{147}-\mathrm{S}-\mathrm{C}_{8} \mathrm{H}_{8}-\mathrm{S}\right]-$ system.

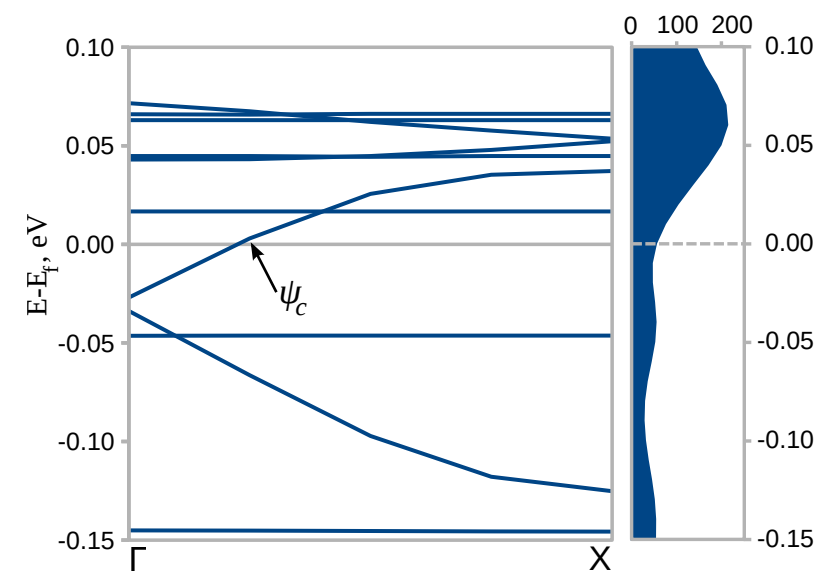

FIG. 2. Band structure (left) and DOS (right) of periodic - $\left[\mathrm{Au}_{147^{-}}\right.$ $\left.\mathrm{S}-\mathrm{C}_{8} \mathrm{H}_{8}-\mathrm{S}\right]$ - system. Fermi level is set to 0 . Symbol $\Psi_{c}$ denotes the position of the electronic state in the conductive band, for which the electron density is shown in Fig. 1

nanorods with a pentagonal section of $\sim 5 \AA$ radius were considered as electrodes. $T(E)$ was calculated with zero potential bias at the electrodes, see Fig.4. The resulting $T(E)$ function at low voltage is obtained as $\simeq 1$, which confirms that the electron can easily transfer from one NP to the other.

\section{PLASMON CALCULATION MODEL}

To build the plasmon model, it is desirable to construct a differential equation of harmonic oscillations, like in the model for bulk plasmons (1). However, while in the latter case the source of the restoring force is the homogeneous polarization and surface charge density, in NP-COM systems the restoring force arises due to the electric field between the two nanoparticles having opposite charges and due to the electrochemical potential $\mu$ difference of the nanoparticles. In the proposed hybrid model it is desirable to use the results of calculations of conductive properties of the NP-COM-NP sys- tems on the basis of the parameters derived from quantumchemical calculations. Furthermore, the existence of a few number of quantum conductive channels in $\mathrm{COM}$ and a huge number of electronic states in the vicinity of $E_{F e r m i}$ of NPs must be taken into account.

The model is based on the assumption that the total energy of the NP-COM-NP system is constant so that

$$
\frac{d E_{t o t}}{d t}=\frac{d\left(E_{t o t_{1}}+E_{t o t_{2}}+E_{t o t_{3}}\right)}{d t}=0,
$$

where $E_{t o t_{1}}, E_{\text {tot }_{2}}, E_{\text {tot }_{3}}$ are the total energies of the first and the second nanoparticles and the bridge molecule between them, correspondingly. These energies can be changed with charge variation in time $E_{\text {tot }_{i}}=E_{\text {tot }_{i}}\left(Q_{i}(t)\right)$, where $i=1 \ldots 3$. We also assume that the charge distribution inside both nanoparticles correspond to the ground states, so from (5) follows

$$
\frac{d E_{t o t_{i}}}{d t}=\frac{d E_{t o t_{i}}}{d Q_{i}} \frac{d Q_{i}}{d t} i=1 \ldots 2
$$

Geometry optimization end calculations of electronic properties of a family consisting of six icosahedron shaped similar gold nanoparticles consisting of 55, 147, 309, 561, 923 and 1415 atoms, see Fig.5, were carried out by the DFTB method $^{52}$ with use of a parameters set that is appropriate for the description of bulk gold clusters and bulk material, as well as $\mathrm{Au}_{n} \mathrm{SCH}_{3}$ clusters ${ }^{53}$. A calculation of the band structure for the periodical structure $-\left[\mathrm{Au}_{147} \mathrm{SC}_{8} \mathrm{H}_{8} \mathrm{~S}\right]$ - was also made. Furthermore, for using in (6) the total energies $E_{t o t}$ of the isolated gold nanoparticles having different total charges $Q(e) \in\{-2,-1,0,1,2\}$ were calculated (Table I).

It was found that the total energy of the all considered nanoparticle is well approximated by quadratic function of the charge (Fig. 6), i.e.

$$
E_{t o t}=a Q^{2}+b Q+c
$$

The coefficients of determination $R^{2}$ in all cases were higher than 0.9999. In these quadratic functions the coefficient $c$ is the total energy and the coefficient $b$ is the opposite value of the Fermi energy of the corresponding neutral nanoparticle correspondingly, see Tables I and II. According to the well-known formula of the charged sphere electrostatic

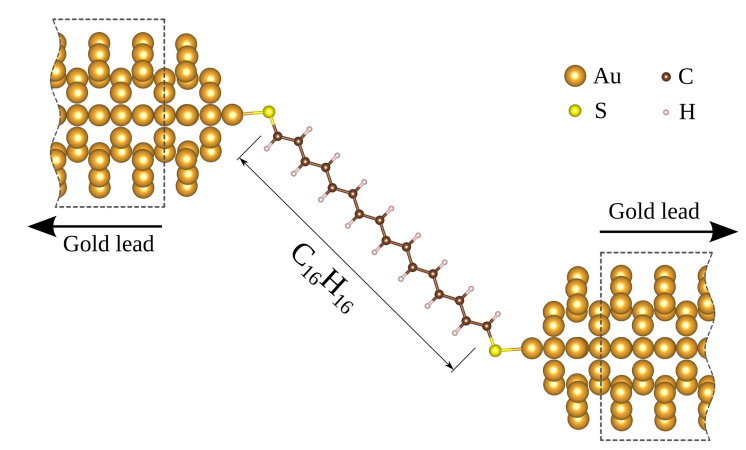

FIG. 3. Geometry of NP-COM-NP system for transmission coefficient calculation. 
TABLE I. Total energies $E_{t o t}$ and Fermi levels $E_{f}$ of neutral and charged gold nanoparticles (everything is in a.u.)

\begin{tabular}{|c|c|c|c|c|c|c|}
\hline & & & \multicolumn{2}{|c|}{$\mathrm{Au}_{309}$} \\
\hline & $E_{t o t}$ & $E_{f}$ & $E_{t o t}$ & $E_{f}$ & $E_{t o t}$ & $E_{f}$ \\
\hline & -156.810 & -0.384 & 20.710 & -0.313 & 85.377 & -0.29 \\
\hline & & & & & & \\
\hline & & & & & & \\
\hline & 559 & -0.1 & & & 029 & \\
\hline & 30 & -0.6 & & & -886.147 & -0.0 \\
\hline & \multicolumn{2}{|c|}{$\mathrm{Au}_{561}$} & \multicolumn{2}{|c|}{$\mathrm{Au}_{923}$} & \multicolumn{2}{|c|}{$\mathrm{Au}_{1415}$} \\
\hline & $E_{t o t}$ & 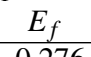 & & 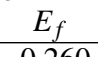 & & $E_{f}$ \\
\hline & & & & 026 & & -0.2 \\
\hline & 608 & & 7 & -0.2 & -40 & -0.22 \\
\hline & 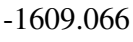 & & & & -405 & -0.1 \\
\hline & & & & & & \\
\hline & & & & & & \\
\hline
\end{tabular}

TABLE II. Coefficients of the quadratic dependence $E=f(Q)$ and nanoparticles radii (everything is in a.u.)

\begin{tabular}{cccccc}
\hline Cluster & $a$ & $b$ & $c$ & $r_{\max }$ & $r_{\min }$ \\
\hline $\mathrm{Au}_{55}$ & 0.0447 & 0.205 & -157.399 & 7.55 & 10.17 \\
$\mathrm{Au}_{147}$ & 0.0320 & 0.182 & -421.201 & 11.41 & 15.49 \\
$\mathrm{Au}_{309}$ & 0.0249 & 0.193 & -885.861 & 15.33 & 20.81 \\
$\mathrm{Au}_{561}$ & 0.0206 & 0.195 & -1609.066 & 19.18 & 26.12 \\
$\mathrm{Au}_{923}$ & 0.0172 & 0.190 & -2647.884 & 23.42 & 31.43 \\
$\mathrm{Au}_{1415}$ & 0.0149 & 0.192 & -4059.705 & 27.36 & 36.78 \\
\hline
\end{tabular}

energy $\left(E=Q^{2} /(2 C)\right.$ ) the coefficient $a$ makes it possible to determine the capacities $C$ of the considered Au clusters as $C=1 /(2 a)$.

In Fig. 7 the dependence of the capacitance $C$ for gold nanoparticles on their radius is shown. It is obvious that this dependence is linear. Since it is not clear how to define the radius of a nanoparticle, two dependencies are presented - on the maximal $r_{\max }$ and on the minimal $r_{\text {min }}$ radius (Table II). The first one is taken as a distance to the atom at the cluster vertex; the second is a distance from the cluster central atom to the cluster faces. The coefficients of $R^{2}$ were in both cases were higher than 0.9998 . The linear coefficients of the straight lines (1.13 and 0.84) in Fig. 7 show in both cases that for some average radius $R_{\text {aver }} \in\left[r_{\min } \ldots r_{\text {max }}\right]$ of the particle its capacitance will coincide with the results of the classical

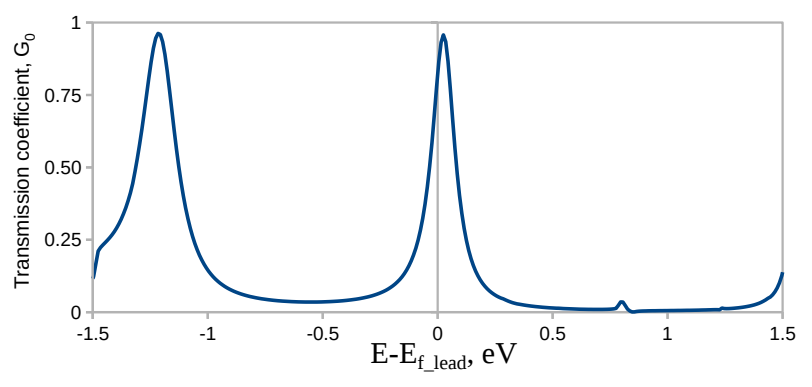

FIG. 4. Transmission coefficient spectrum (in units of $2 e^{2} / h$ ) of NPCOM-NP system. The energy is 58 measured with respect to the Fermi level of the leads.

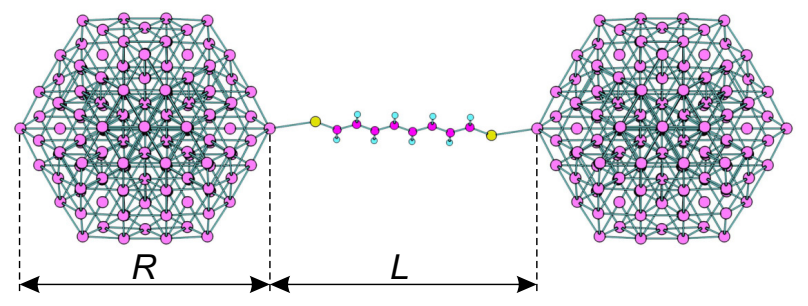

FIG. 5. Geometry of $\mathrm{Au}_{147}-\mathrm{S}-\mathrm{C}_{8} \mathrm{H}_{8}-\mathrm{S}-\mathrm{Au}_{147}$ system.

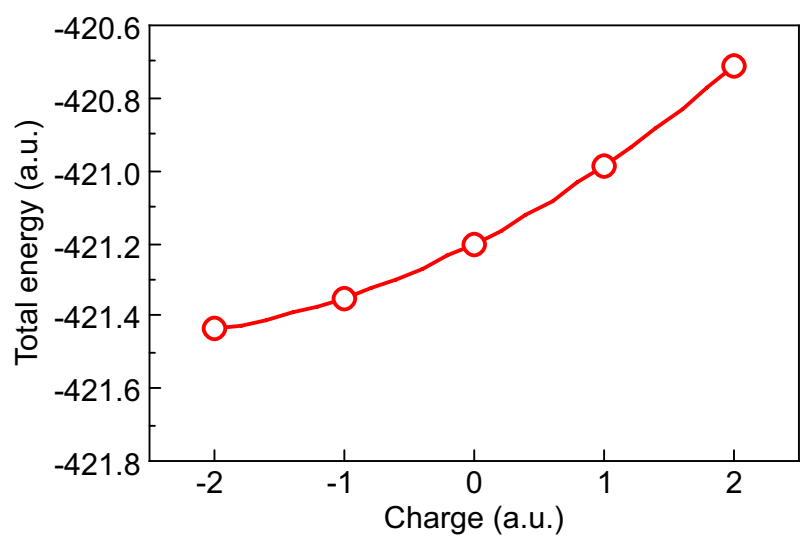

FIG. 6. Dependence of the $\mathrm{Au}_{147}$ nanoparticle total energy on the charge.

electrostatics $C=R$, i.e. the capacitance of the nanoparticle is equivalent to that of a conducting sphere and equal to the average nanoparticle radius $R_{\text {aver }}$. We speculate that the existence of free terms in both linear dependencies, which do not change much, is related to the bulk distribution of the extra charge inside the particle. In any case, this contribution is significantly smaller than the surface contribution, which suggests that additional charge is almost completely distributed over the nanoparticle surface.

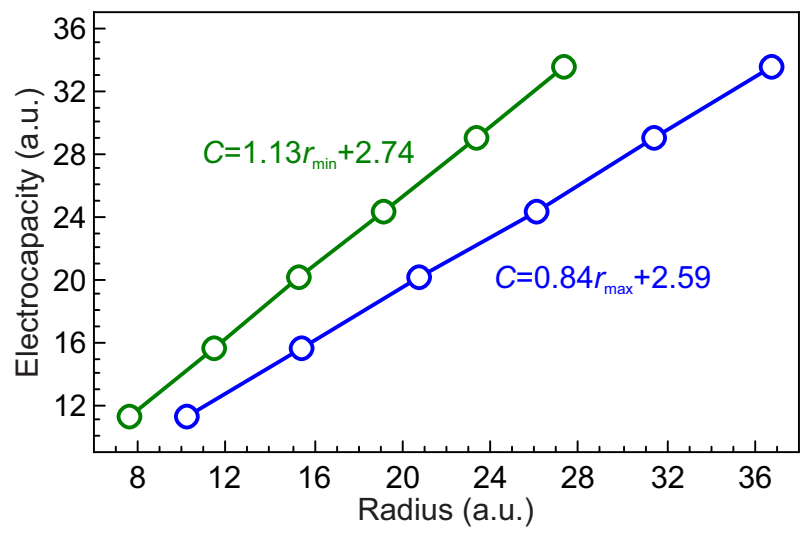

FIG. 7. Dependence of the Au nanoparticles capacitance on their radius.

Equation (7) also assumes that extra-charge in the particle is distributed according the ground state. Actually, relaxation of non-equilibrium electrons occurs with a frequency $v \approx \tau^{-1}$ 
where $\tau^{-1}$ is the reciprocal time between electron collisions. For small nanoparticles this time is even reduced due to scattering at the nanoparticle boundaries. For gold $\tau \sim 29 \mathrm{fs}^{56}$ and we assume that the frequency of the plasmons under consideration is much less than this inverse value, so the nanoparticles having a varying charge can be considered to be in the ground state under the plasmon oscillations.

The dependency of the total energy of the two isolated nanoparticles with index $i E_{\text {tot }_{i}}\left(Q_{i}\right)$ were calculated as described in the previous section, see (7), where $b_{i}$ denote the nanoparticle chemical potentials. Using the fact that the volumes of both nanoparticles are much larger than the volume of the conductive molecule, most likely the electronic density of the states (DOS) at the Fermi level $E_{f}$ for the two nanoparticles $\operatorname{DOS}\left(E_{f}\right)_{\mathrm{NP}_{1}}$ and $\operatorname{DOS}\left(E_{f}\right)_{\mathrm{NP}_{2}} \gg \operatorname{DOS}\left(E_{f}\right)_{\mathrm{COM}}$. It means that with the change of $E_{f}$ the change of cluster charges follow $\Delta Q_{\mathrm{NP}_{1}}$ and $\Delta Q_{\mathrm{NP}_{2}} \gg \Delta Q_{\mathrm{COM}}$ and that during the plasmon oscillations the charges $Q_{i}(t)$ of both nanoparticles are opposite and the conducting molecule would stay neutral. Following this situation and assuming that the two nanoparticles are identical, one gets

$$
\frac{d\left(E_{t o t_{1}}+E_{t o t_{2}}\right)}{d t}=\left[\left(b_{1}-b_{2}\right)+\left(a_{1}+a_{2}\right) 2 Q_{1}\right] \frac{d Q_{1}}{d t},
$$

where $Q_{1}=Q_{1}(t)$ is the charge of the first nanoparticle. Furthermore, by virtue of the argument stated above, this charge of the nanoparticle will be denoted $Q(t)$ and the derivative $\frac{d Q_{1}(t)}{d t}$ will be denoted as a current

$$
\frac{d Q_{1}(t)}{d t} \equiv I(t) .
$$

This implies that the electron freely moves from one particle through the conducting molecule to the other particle. In reality, it is necessary to take into account and determine the transmission probability $\mathrm{T}$ as the probability for an electron to transmit through the bridge multiplied by the number of transverse modes, each of which transfers electron through the bridge. Using the definition data, the total energy of the two isolated nanoparticles in (8) can be rewritten as

$$
\frac{d\left(E_{t o t_{1}}+E_{t o t_{2}}\right)}{d t}=4 a Q(t) I(t) .
$$

Unfortunately, in (5-10) it was assumed the two nanoparticles do not influence each other. In contrast, due to the electrostatic interaction their total energy is rewritten as

$$
\begin{aligned}
\frac{d E_{t_{\text {tot }}-2}}{d t} & =4 a Q(t) I(t)-\frac{d}{d t}\left[F(R, L) \frac{Q(t)^{2}}{2 R+L}\right]= \\
& =Q(t) I(t)\left(4 a-2 \frac{F(R, L)}{2 R+L}\right),
\end{aligned}
$$

where the second term on the right side equation corresponds to the derivative of the electrostatic interaction of the two opposite charges at a distance $2 R+L$ between the NP centers. A correction function $F(R, L)$ is introduced to take into account the difference between the interaction of the two conducting spheres (nanoparticles) and point charges at the polarized spheres. The function $F(R, L)$ was addressed in ${ }^{57,58}$, where it was shown that this function decreases rapidly from 2.0 when two conducted spheres are touching to 1.0 when $\frac{L}{2 R} \rightarrow \infty$ and $\left.F(R, L)\right] \simeq 1.07$ at $\frac{L}{2 R}=1.4^{58}$. Next, the force acting on the conduction electron(hole) inside the conducting molecule is obtained as

$$
\begin{aligned}
& F(x, t)=-e E(x, t)-e[\vec{v} \vec{B}], \\
& E(x, t)=-\nabla \phi(x, t)-\frac{\partial A}{\partial t} .
\end{aligned}
$$

We recall that for one-dimensional carriers moving in the $\mathrm{COM}$, the Lorentz magnetic force in the second right term of (12) should be neglected.

Assuming that the characteristic size $\tilde{R}$ of the system is much smaller than the wavelength of the electromagnetic wave or $\tilde{R} \ll c T$, where the $T$ - period of EMF, $c$ - light velocity, the quasistatic approximation (QSA) may be used in the model. It means that EMF delay effects can be neglected. In QSA the characteristic speed of electrons are much smaller then $c(v \ll c)$ and the bias current is much smaller than the conduction current: $\left|\frac{\partial D}{\partial t}\right| \ll|j|$ in QSA $\Delta A(\vec{x}, t)=-\frac{4 \pi}{c} I(x, t)$, where $I(x, t)$ is the current at coordinate $x$ passing through the $\mathrm{COM}$ at the time $t$. By estimation, taking into account the speed of light in the atomic system of units $C \simeq 137$ and the magnitude of the current $I(x, t) \approx \frac{e}{L} v_{\text {Fermi }}$, the second term in the right part of (12) can be neglected and the force acting on the carriers can be defined as the gradient of the electrostatic potential $\nabla \phi(x, t)$ only. Further, in QSA the current reads

$$
j=\sigma E, \operatorname{div}(j) \approx 0,
$$

so it is possible to disregard the inhomogeneity of the current inside the conductive molecule and $I(x, t)=I(t)$.

Opposite to the Landauer approach, where the free electron or hole dynamics inside the COM of NP-COM-NP system under EMF is not analyzed, we have to do just that for building the plasmon differential equation. As it was mentioned above, it is assumed that this carrier dynamics is similar to the dynamics in periodical -(NP-COM)- systems under the same EMF. Assuming that the EMF is sufficiently small for Zener or avalanche breakdown, the electrons (holes) will be accelerated according to the effective mass tensor near the Fermi level. Due to 1D nature of the carrier movement this tensor transforms to the carrier effective mass value

$$
m^{*}=\hbar^{2}\left[\frac{d^{2} E}{d k^{2}}\right]_{k=k_{f}}^{-1} .
$$

In the following text we will build our model in relation to the periodic - $\left[\mathrm{Au}_{147}-\mathrm{S}-\mathrm{C}_{8} \mathrm{H}_{8}-\mathrm{S}\right]-$ system. According to Fig.2, the system is metallic. The calculated effective mass value $m^{*}=-0.454 m_{e}$ was calculated for the system using (14). Due to the system symmetry and the symmetry $(k \Rightarrow-k)$, the total current of the free electrons and holes near $E_{\text {fermi }}$ is cancelled without any AC electromagnetic field. In other words, the linear dependence of energy $E(k)$ is insignificant and the parabolic dependence

$$
E(k)=\frac{(\hbar k)^{2}}{2 m^{*}}
$$


should be used for the carriers. We recall that the COM stays neutral under plasmon generation, so the total number of carriers inside the COM does not change under an applied weak EMF. The COM total energy $E_{\text {tot }_{3}}$ can be written via a sum of electrons in the conduction band, having different quasimomentum (15)

$$
E_{\text {tot }_{3}}=\sum_{i} n_{i} \frac{\hbar^{2} k_{i}^{2}}{2 m^{*}}
$$

where $n_{i^{-}}$occupation degrees of electrons having quasimomentum $k_{i}$.

Taking into account that under the influence of a weak electric field the carriers are excited only near the Fermi level, we can write the energy derivative

$$
\frac{d E_{t o t_{3}}}{d t}=n_{f} \frac{\hbar k}{m^{*}}\left[\frac{d(\hbar k)}{d t}\right]_{k=k_{f}},
$$

where $n_{f}$ - occupation degrees of electrons having Fermi quasi-momentum $k=k_{f}$. Due to spin degeneracy and generation of holes with the same effective mass upon excitation of electrons near the Fermi level, in the future we will consider only electrons for which $n_{f}=4$.

Following Ref. ${ }^{43}$ and equations $(2,3,15)$, the current and its derivative can be expressed as

$$
\begin{aligned}
I(t) & =\frac{-e n_{f}}{L} \frac{1}{\hbar} \frac{\partial E(k)}{\partial k}= \\
& =\left.\frac{-e n_{f}}{L} \frac{\hbar k}{m^{*}}\right|_{k=k_{f}} \\
\frac{d I(t)}{d t} & =\left.\frac{-e n_{f}}{L m^{*}} \frac{d(\hbar k)}{d t}\right|_{k=k_{f}},
\end{aligned}
$$

where $L$ - length of COM.

Combining (16-18), the $E_{\text {tot }_{3}}$ derivative is equal to

$$
\frac{d E_{t o t_{3}}}{d t}=I(t) \frac{d I(t)}{d t} \frac{m^{*} L^{2}}{n e^{2}} .
$$

Using $(9,11,19)$, equation (5) is transformed into

$$
\begin{array}{r}
\frac{d E_{t o t}}{d t}=Q(t) I(t)\left(4 a-2 \frac{F(R, L)}{2 R+L}\right)+ \\
+I(t) \frac{d^{2} Q(t)}{d t^{2}} \frac{m^{*} L^{2}}{n e^{2}}=0 .
\end{array}
$$

Dividing by $I(t)$ one can get a differential equation of the harmonic oscillations having the square of modified plasmonic frequency $\widetilde{\omega_{p l}^{2}}$

$$
\begin{aligned}
\frac{d^{2} Q(t)}{d t^{2}} & =-\widetilde{\omega_{p l}^{2}} Q(t) \\
\widetilde{\omega_{p l}^{2}} & =\left(\frac{1}{C}-\frac{F(R, L)}{2 R+L}\right) \frac{2 n e^{2}}{m^{*} L^{2}} .
\end{aligned}
$$

Here substitution $C=1 /(2 a)$ is used again. From this equation one can see that the square of modified plasmonic frequency $\widetilde{\omega_{p l}^{2}}$ is similar with expression of conventional plasma frequency $\omega_{p l}^{2}=4 \pi n e^{2} /\left(m^{*} \Omega\right)$, where $n$ denotes the number of electrons occupying the volume $\Omega$. Under the assumption of $R \gg L$, remembering that $C \cong R$ (NP radius) and replacing the expression in parentheses of (22) by $1 / R$, one gets

$$
\widetilde{\omega_{p l}^{2}} \approx \frac{2 n e^{2}}{m^{*} L^{2} R}=\frac{4 \pi n e^{2}}{m^{*} \widetilde{\Omega}},
$$

where $\widetilde{\Omega}=2 \pi R L^{2}$ - effective volume per $n$ electrons. Comparing $\widetilde{\omega_{p l}^{2}}$ and $\omega_{p l}^{2}$ we can verify that the modified plasma frequency resides in the infrared (IR) region. For example, in the bulk gold one electron has a volume of $16.85 \AA^{3}$ and the plasmonic frequency $\omega_{p l} i s \approx 9.1 \mathrm{eV}$. Using the parameters of the investigated system containing the nanoparticle of 147 gold atoms $(\langle R\rangle \cong 14.0 \AA, L \cong 14.3 \AA$, the estimation of the modified plasma frequency is $\widetilde{\omega_{p l}} \approx 0.35 \mathrm{eV}$.

In order to show the influence of system geometric parameters $(\mathrm{R}, \mathrm{L})$ on the plasmon frequency, we plotted $\widetilde{\omega_{p l}}$ as a function of the nanoparticle radius $\mathrm{R}$ for three conducting molecules -[S- $\left.\mathrm{C}_{n} \mathrm{H}_{n}-\mathrm{S}\right]-(\mathrm{n}=6,7,8)$, having different length $\mathrm{L}$ (see Fig.8 and legend there). The electron effective mass was here taken again as $m^{*}=0.454 m_{e}$.

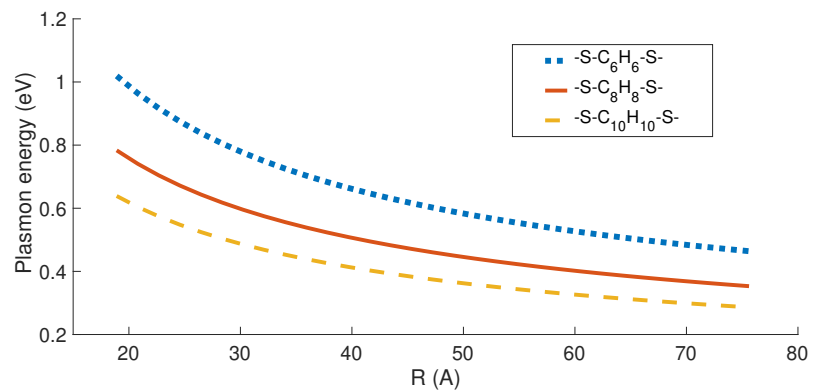

FIG. 8. The dependence of plasmon frequency of nanoparticle on its radius $\mathrm{R}$ for three conducting molecules $-\left[\mathrm{S}-\mathrm{C}_{n} \mathrm{H}_{n}-\mathrm{S}\right]-(\mathrm{n}=6,7,8)$.

One can see that in the proposed NP-COM-NP systems the plasmonic frequencies are expected to lie in IR region and are very significantly changed with a change of the system geometric parameters.

\section{CONCLUSION}

In this work the possibility of generating specific plasmons due to charge transfer in a couple of metal nanoparticles bridged by conductive molecules is investigated. To describe the properties of such plasmons, an original quantumclassical model was developed based on a description of the time dependence of the ballistic current through the conductive bridge and which includes quantum effects. For the test system, consisting of two gold nanoparticles bridged by the conjugated poly-acetylene molecule $\mathrm{C}_{8} \mathrm{H}_{8}$ terminated by sulfur atoms, an analytical expression for the frequency of the plasmons was derived. Our approach can be qualified from the fact that although approaches based on calculations of non-equilibrium Green functions or real-time propagation of 
wave functions in principle can be applied to describe ballistic transport, their complexity disallows the description of real sophisticated systems of the kind considered here. The model proposed in this work resolves this problem since it uses only band structure information which can be easily obtained from the calculations of corresponding periodical system, whose unit cell consists of the connected nanoparticle and the conductive molecule. It was shown that for the systems under study the plasmon frequency is determined by the expression (23), which is similar to the one for bulk materials. Herewith, the effective density of the conduction electrons becomes in the present case significantly lower than the density of conduction electrons in the bulk material. It results in shifting the modified plasmon frequency to the IR region. The strong dependence of the modified plasmon frequency on the system conductivity makes it possible to use such NP-COM-NP systems to build different chemical sensors which can be based on a change in the conductivity of the conducting molecule during its chemical interaction with external molecules. Such an interaction can thus significantly change the conjugated character of the $\pi$-bonding in the molecule and, therefore, its conductivity. The conductivity directly changes the effective mass and, according to (22), the plasmon frequency, that can be easily measured. We believe that this new type of plasmons can have a unprecedented impact on the field of deep tissue chemical sensing.

Acknowledgments: This study was supported by the Russian Science Foundation, project no. 18-13-00363.

${ }^{1}$ A. Uddin and X. Yang, J. Nanosci. Nanotechnol. 14, 1099 (2014).

${ }^{2}$ J. Liu, H. He, D. Xiao, S. Yin, W. Ji, S. Jiang, D. Luo, B. Wang, and Y. Liu, Materials 11, 1833 (2018).

${ }^{3}$ J. Olson, S. Dominguez-Medina, A. Hoggard, L.-Y. Wang, W.-S. Chang, and S. Link, Chem. Soc. Rev. 44, 40 (2015).

${ }^{4}$ L. Guo, J. A. Jackman, H.-H. Yang, P. Chen, N.-J. Cho, and D.-H. Kim, Nano Today 10, 213 (2015).

${ }^{5}$ A. N. Koya and J. Lin, Appl. Phys. Rev. 4, 021104 (2017).

${ }^{6}$ N. Elahi, M. Kamali, and M. H. Baghersad, Talanta 184, 537 (2018).

${ }^{7}$ Z. Farka, T. Juř́ík, D. Kováŕ, L. Trnková, and P. Skládal, Chem. Rev. 117, 9973 (2017).

${ }^{8}$ K. A. Willets, A. J. Wilson, V. Sundaresan, and P. B. Joshi, Chem. Rev. 117, 7538 (2017).

${ }^{9}$ C. Deeb and J.-L. Pelouard, Phys. Chem. Chem. Phys. 19, 29731 (2017).

${ }^{10}$ S. Linic, U. Aslam, C. Boerigter, and M. Morabito, Nat. Mater. 14, 567 (2015).

${ }^{11}$ M. Valenti, M. P. Jonsson, G. Biskos, A. Schmidt-Ott, and W. A. Smith, J. Mater. Chem. A 4, 17891 (2016).

${ }^{12}$ A.E. Ershov, V.S. Gerasimov, A.P. Gavrilyuk, S.V. Karpov, V.I. Zakomirnyi, I.L. Rasskazov, and S.P. Polyutov, Journal of Quantitative Spectroscopy and Radiative Transfer, 191, 1-6 (2017)

${ }^{13}$ I.L. Rasskazov, S.V. Karpov, V.A. Markel, Opt. Lett., 38, iss. 22, 47434746 (2013)

${ }^{14}$ I.L. Rasskazov, S.V. Karpov, G.Y. Panasyuk, and V.A. Markel, Journal of Applied Physic, 119, iss. 4, 043101 (2016)

${ }^{15}$ V.I. Zakomirnyi, I.L. Rasskazov, V.S. Gerasimov, A.E. Ershov, S.P. Polyutov, S.V. Karpov, H. Ågren, Photonics and Nanostructures - Fundamentals and Applications, 30, 50-56 (2018)
${ }^{16}$ V.I. Zakomirnyi, I.L. Rasskazov, S.V. Karpov, S.P. Polyutov, Journal of Quantitative Spectroscopy and Radiative Transfer, 187, 54-61 (2017)

${ }^{17}$ V.I. Zakomirnyi, I.L. Rasskazov, V.S. Gerasimov, A.E. Ershov, S.P. Polyutov, S.V. Karpov, Applied Physics Letters, 111, iss. 12, 123107 (2017)

${ }^{18}$ N. Venugopal, A.E. Gerasimov, V.S., Ershov, S.V. Karpov, S.P. Polyutov, Optical Materials, 72, 397-402 (2017)

${ }^{19}$ F. Parveen, B. Sannakki, M. V. Mandke, and H. M. Pathan, Sol. Energy Mater. Sol. Cells 144, 371 (2016).

${ }^{20}$ S. Gwo, H.-Y. Chen, M.-H. Lin, L. Sun, and X. Li, Chem. Soc. Rev 45, $5672(2016)$.

${ }^{21}$ K. Ueno, T. Oshikiri, Q. Sun, X. Shi, and H. Misawa, Chem. Rev. 118, 2955 (2018).

${ }^{22}$ M. Ujihara, J. Oleo Sci. 67, 689 (2018).

${ }^{23}$ S. Lee and I. Choi, Biochip J. 13, 30 (2019).

${ }^{24}$ A. Crut, P. Maioli, F. Vallée, and N. D. Fatti, J. Phys.: Condens. Matter 29, 123002 (2017).

${ }^{25}$ R. Esteban, A. G. Borisov, P. Nordlander, and J. Aizpurua, Nat. Commun. 3, 825 (2012).

${ }^{26}$ R. Esteban, A. Zugarramurdi, P. Zhang, P. Nordlander, F. J. Garcia-Vidal, A. G. Borisov, and J. Aizpurua, Faraday Discuss. 178, 151 (2015).

${ }^{27}$ W. Zhu, R. Esteban, A. G. Borisov, J. J. Baumberg, P. Nordlander, H. J. Lezec, J. Aizpurua, and K. B. Crozier, Nat. Commun. 7, 11495 (2016).

${ }^{28}$ P. Hohenberg and W. Kohn, Phys. Rev. 136, B864 (1964).

${ }^{29}$ W. Kohn and L. J. Sham, Phys. Rev. 140, A1133 (1965).

${ }^{30}$ E. Runge and E. K. U. Gross, Phys. Rev. Lett. 52, 997 (1984).

${ }^{31}$ X. L. Lozano, C. Mottet, and H.-Ch. Weissker, J. Phys. Chem. C 117, 3062 (2013).

${ }^{32}$ V. Kulkarni, E. Prodan, and P. Nordlander, Nano Lett. 13, 5873 (2013).

${ }^{33}$ E. Townsend and G. W. Bryant, Nano Lett. 12, 429 (2012).

${ }^{34}$ X. López-Lozano, H. Barron, C. Mottet, and H.-C. Weissker, Phys. Chem. Chem. Phys. 16, 1820 (2014).

${ }^{35}$ V. Kulkarni and A. Manjavacas, ACS Photonics 2, 987 (2015).

${ }^{36}$ Electrons and Phonons. The theory of transport phenomena in solids. J. M. Ziman. Oxford University Press, New York, 1960.

${ }^{37}$ A. Varas, P. García-González, J. Feist, F. J. García-Vidal, and A. Rubio, J. Nanophotonics 5, 409 (2016).

${ }^{38}$ X. López-Lozano, H. Barron, C. Mottet, and H.-C. Weissker, Phys. Chem. Chem. Phys. 16, 1820 (2014).

${ }^{39}$ G. Kresse and J. Furthmöller, Comput. Mater.Sci. 6, 15 (1996).

${ }^{40} \mathrm{G}$. Kresse and J. Furthmöller, Phys. Rev. B 54, 11169 (1996).

${ }^{41}$ M. Sukharev and M. Galperin, Phys. Rev. B, 81, 165307 (2010).

${ }^{42}$ R. Landauer, Philos. Mag. 21, 863 (1970).

${ }^{43}$ S. Datta, Quantum Transport: Atom to Transistor, Cambridge University Press, New York, 2005

${ }^{44}$ L. V. Keldysh, JETP 20, 1018 (1965).

${ }^{45}$ L. P. Kadanoff, and G. Baym, Quantum Statistical Mechanics, Frontiers in Physics Lecture Notes, Benjamin/Cummings (1962).

${ }^{46} \mathrm{G}$. Stefanuci, and R. van Leewuen, Non-Equilibrium Many-Body Theory of Quantum Systems, Cambridge (2013).

${ }^{47}$ www.openmx-square.org

${ }^{48}$ U. Peskin, Fortschr. Phys. 65, 1600048 (2017).

${ }^{49}$ M. Sukharev, M. Galperin, Phys. Rev. B 81, 165307 (2010).

${ }^{50}$ A. J. White, M. Sukharev and M. Galperin, Phys. Rev. B 86, 205324 (2012).

${ }^{51}$ M. Kuperman and U. Peskin, Molecular Electronics: A Theoretical and experimental Approach, Taylor \& Francis, 2015.

${ }^{52}$ B. Aradi, B. Hourahine, and T. Frauenheim, J. Phys. Chem. A, 111, 5678 (2007).

${ }^{53}$ A. Fihey, C. Hettich, J. Touzeau, F. Maurel, A. Perrier, C. Köhler, B. Aradi, and T. Frauenheim, J. Comp. Chem. 36, 2075 (2015).

${ }^{54}$ F. Alkan and C.M. Aikens, J. Phys. Chem. C 122, 23639 (2018).

${ }^{55}$ N.V. Ilawe, M.B. Oviedo, and B.M. Wong, J. Mater. Chem. C 6, 5857 (2018).

${ }^{56}$ P. B. Johnson and R. W. Christy, Phys. Rev. B 6, 4370 (1972).

${ }^{57}$ W. R. Smythe, Static and Dynamic Electricity, McGraw-Hill Book Company, Inc. (1950).

${ }^{58}$ V. A. Saranin, Physics-Uspekhi 42, 385 (1999). 